\title{
Grau de complexidade dos cuidados de enfermagem: readmissões hospitalares de pessoas com câncer de mama
}

\author{
Degree of complexity of nurse care: associations in clinical oncology in breast cancer \\ Grado de complejidad de los cuidados de enfermería: asociaciones en oncología clínica en \\ cáncer de mama

\section{Lívia Gomes da Silva ${ }^{a}$ Marléa Chagas Moreirab}

\section{Como citar este artigo:}

Silva LG, Moreira MC. Grau de complexidade dos cuidados de enfermagem: readmissões hospitalares de pessoas com câncer de mama. Rev Gaúcha Enferm. 2018;39:e20180015. doi: https://doi. org/10.1590/1983-1447.2018.20180015
`Instituto Nacional de Câncer (INCA), Unidade 3. Rio de Janeiro, Rio de Janeiro, Brasil.

- Universidade Federal do Rio de Janeiro (UFRJ), Escola de Enfermagem Anna Nery. Rio de Janeiro, Rio de Janeiro, Brasil.

\section{RESUMO}

Objetivo: Avaliar o grau de complexidade dos cuidados de enfermagem de pacientes com câncer de mama readmitidas na oncologia clínica.

Métodos: Estudo transversal com 108 pessoas com câncer de mama readmitidas na oncologia clínica num centro de alta complexidade de oncologia do Rio de Janeiro durante 2015. Realizada análise documental sendo dados analisados estatisticamente.

Resultados: Os graus de complexidade de cuidados de enfermagem predominantes foram semi-intensivos (36,1\%) e cuidados intensivos (36,1\%). Na análise multivariada, apenas a performance status $(p<0,001)$ e a hipertensão arterial $(p=0,024)$ permaneceram associados ao grau de complexidade.

Conclusões: 0 grau de complexidade dos pacientes readmitidos foi predominantemente semi-intensivo e intensivo. Essa avaliação implica no gerenciando do cuidado por meio do conhecimento do perfil dos pacientes com câncer de mama em readmissão hospitalar e da detecção das características associadas ao grau de complexidade.

Palavras-chave: Enfermagem oncológica. Readmissão do paciente. Determinação de necessidades de cuidados de saúde. Neoplasias da mama.

\section{ABSTRACT}

Objective: It was to evaluate the degree of complexity of nursing care required for breast cancer patients who are readmitted to hospital.

Methods: Cross-sectional study whose 108 subjects were breast cancer patients in unplanned readmission into the oncological unit of hospital in Brazil. Used for documentary analysis and the data analyzed statistically.

Results: The predominant degrees of complexity of complexity of nursing care were semi-intensive (36.1\%) and intensive care (36.1\%). In the multivariate analysis, only performance status $(p<0.001)$ and arterial hypertension $(p=0.024)$ remained associated with the degree of complexity

Conclusions: The degree of complexity of patients readmitted was predominantly semi-intensive and intensive. This evaluation implies in the management of the care by means of the knowledge of the the profile breast cancer patients in hospital readmission and the detection of the characteristics associated to the degree of complexity.

Keywords: Oncology nursing. Patient readmission. Needs assessment. Breast neoplasms.

\section{RESUMEN}

Objetivo: Evaluar el grado de complejidad de los cuidados de enfermería en pacientes con cáncer de mama readmitidas en la oncología clínica.

Métodos: Estudio transversal con 108 personas con cáncer de mama readmitidas en la oncología clínica de un centro de alta complejidad en oncología durante el año 2015. Realizado análisis documental siendo datos fueron analizados estadísticamente.

Resultados: los grados de complejidad de los cuidados intensivos (36,1\%) y cuidados intensivos (36,1\%). En análisis multivariado apenas PS performance status $(p<0,001)$ y la hipertensión arterial permanecieron con asociación estadística al grado de complejidade. Conclusiones: el grado de complejidade de los pacientes readmitidos fue predominantemente semi-intensivo y intensivo. Esta evaluación implica la gestíon de los cuidados mediante el conocimiento del perfil de los pacientes con cáncer de mama en la readmisión hospitalaria y de la deteccíon de las características asociadas al grado de complejidad.

Palabras clave: Enfermería oncológica. Readmisión del paciente. Evaluación de necesidades. Neoplasias de la mama. 


\section{—INTRODUÇÃO}

A classificação de pacientes de acordo com seu grau de complexidade é uma estratégia de avaliação capaz de contemplar as reais necessidades dos pacientes. Para sua implementação, recomenda-se o uso de um sistema de classificação de pacientes a fim de identificar sistemática e efetivamente tais necessidades ${ }^{(1)}$.

O câncer de mama tem grande magnitude no que tange a saúde pública no Brasil e no mundo ${ }^{(2)}$ e a assistência de enfermagem a pacientes acometidos por tais neoplasias deve estar pautada nas necessidades de saúde desses indivíduos.

Em diversas regiões do Brasil, o câncer de mama ainda é diagnosticado em estádio tardio(3), acarretando implementação de tratamentos mais agressivos que aumentam a morbidade dos pacientes e os expõem a maiores episódios de admissões e readmissões hospitalares para reabilitação da saúde ${ }^{(4)}$.

A readmissão hospitalar tem larga relevância na atenção oncológica. As possíveis complicações do paciente com câncer requerem muitas das vezes uma assistência hospitalar para a reabilitação do seu quadro clínico. Um hospital estadual de Bauru avaliou a readmissão hospitalar e suas possíveis causas e concluiu que estas podem ocorrer por condições ligadas a assistência intra-hospitalar, ao estado clínico do indivíduo e até à questões sociais e ambientais. Verificaram também que muitas dessas readmissões poderiam ser evitadas após um conhecimento prévio de seus fatores associados ${ }^{(5)}$.

A equipe de enfermagem representa um segmento muito importante na assistência hospitalar. A falta de recursos financeiros no setor saúde tem ocasionado uma redução de custos e consequentemente um corte nos recursos humanos, fato esse que implica fortemente na qualidade do cuidado de enfermagem prestado.

Para uma assistência de qualidade, a enfermeira no gerenciamento do cuidado deve ser capaz de oferecer um cuidado sistematizado. Para isso, é primordial o planejamento assistencial adequado voltado às necessidades do indivíduo prevendo os recursos necessários para uma assistência de qualidade e segura(2).

Nessa perspectiva, o Sistema de Classificação de Pacientes é uma estratégia de avaliação que contempla as reais necessidades dos pacientes e subsidia as decisões da enfermeira no gerenciamento do cuidado, a partir do grau de complexidade do cuidado(2).

Nesse sentido, delineou-se esse trabalho com o objetivo de avaliar o grau de complexidade dos cuidados de enfermagem de pessoas com câncer de mama em situação de readmissão hospitalar e sua implicação para o gerenciamento do cuidado de enfermagem.

\section{- MÉTODOS}

Estudo descritivo e retrospectivo com população composta por pacientes com câncer mama readmitidas em até 30 dias após alta na oncologia clínica de um Centro de Alta Complexidade de Oncologia (CACON) da cidade do Rio de Janeiro, Rio de Janeiro, Brasil.

Tal período foi especificado em alinhamento às evidências apresentadas pela Sociedade Americana de Oncologia Clínica em relação à predominância de pesquisas sobre readmissões hospitalares tardias, ou seja, com períodos de até 30 dias após alta. A readmissão tem sido considerada um indicador de qualidade assistencial, o qual possibilita avaliar e repensar o atendimento e planejamento dos cuidados que serão necessários após a alta, identificando fatores evitáveis de alta precoce e evitando uma nova internação não programada ${ }^{(6)}$.

Inicialmente, foi buscado num sistema de informação do cenário do estudo o número de internações na oncologia clínica em 2015. Esses dados foram tabulados no Excel, versão 2010 e a partir daí realizada uma seleção dos registros de pacientes em situações de readmissão hospitalar em até 30 dias após alta através de tabela dinâmica. Essa seleção compôs um total de 131 pessoas. A partir do emprego dos critérios de inclusão e exclusão citados abaixo, ocorreu uma perda de 23 registros. Portanto a população de estudo teve valor igual a 108 casos de readmissão hospitalar em até 30 dias após alta da oncologia clínica.

Os critérios de inclusão foram: pacientes com 18 anos ou mais; presença de registro de autorização de internação hospitalar (AlH) com unidade solicitante designada para oncologia clínica. Já os critérios de exclusão: pacientes que ausência de registro no prontuário físico e nos sistemas de informação correspondente ao primeiro dia da readmissão hospitalar de pessoas com câncer de mama readmitidas na unidade de internação de oncologia clínica em até 30 dias após a alta hospitalar.

Há de destacar que inclusão no estudo de participantes apenas em readmissão hospitalar foi empregada a partir de justificativas de estudos de grau de dependência em unidades de internação que destacam que pacientes com maior número de internações são mais complexos ${ }^{(7)}$.

A técnica de coleta de dados utilizada foi a análise documental. Inicialmente foi realizada coleta de dados no sistema de informação de administração hospitalar, logo após no prontuário eletrônico e por último no prontuário físico dos participantes da pesquisa. 
O instrumento para coleta de dados foi baseado no roteiro estruturado abordando as seguintes variáveis independentes: sexo, idade, estado civil, escolaridade, ocupação profissional principal, renda mensal, nível de atenção na rede assistencial que ocorreu diagnóstico da doença, estadiamento do câncer da mama, Escala Performace Status (PS) de acordo com Escala Zubrod, comorbidades e intervalo da readmissão hospitalar, esse último se até 10 dias ou entre 11 e 30 dias após alta hospitalar. Como variável dependente ou de desfecho, obteve-se o grau de complexidade de cuidados de enfermagem, ou seja, o sistema de classificação de pacientes de Perroca ${ }^{(8)}$, composto por nove indicadores cujo somatório de escores dava o resultado da classificação do grau de complexidade do cuidado obtida.

No cenário de estudo, o PS de acordo com Escala Zubrod é avaliado e registrado no prontuário do paciente pela equipe multidisciplinar durante os atendimentos ambulatoriais e internações. Essa avaliação da capacidade funcional do paciente varia de 0 a 4, na qual 0 é aquele completamente funcional e assintomático e 4 o paciente restrito ao leito, muito doente requerendo assistência ativa ${ }^{(9)}$.

O estadiamento do câncer de mama classifica a extensão da doença por meio das classificações no sistema TNM, obtendo-se um estágio geral que varia entre I, II, III e IV. Os canceres estágio I são os menos avançados e muitas vezes têm melhores prognósticos. Já estágio IV, possui metástase a distância e são mais avançados ${ }^{(10)}$.

O sistema de classificação de pacientes de Perroca compreende nove indicadores que correspondem a áreas especificas de cuidados de enfermagem nas dimensões biológicas e psicossociais dos indivíduos e também aqueles relacionados ao planejamento assistencial. Estes indicadores são: planejamento e coordenação do processo de cuidar; investigação e monitoramento; cuidado corporal e eliminações; cuidados com pele e mucosas; nutrição e hidratação; locomoção e atividade; terapêutica; suporte emocional; educação à saúde. Suas respectivas respostas possuem escores com gradação de 1 a 4 pontos. O grau de complexidade do cuidado é definido pelo somatório desses escores, classificando-se em: cuidados mínimos (9 - 12 pontos), cuidados intermediários (13-18 pontos), cuidados semi-intensivos (19-24 pontos) ou cuidados intensivos (25-36 pontos).

Os dados foram tabulados e analisados no Software estatístico SPSS (Statistical Package for Social Sciences) versão 21.0. Para avaliar a associação entre as variáveis e o desfecho grau de complexidade, primeiramente foi realizada a analise bivariada com o Teste Qui-Quadrado de Pearson e Teste Exato de Fisher. Nessa primeira etapa, o valor $p<0,20$ foi utilizado para indicar quais variáveis seriam utilizadas no teste de associação. Logo após, foi realizada a análise multivariada com as variáveis consideradas com significância estatística e realizado teste de Regressão Logística Binária por meio do método Forward. Como significância estatística, foi tomado valor $p<0,05$ e índice de confiança de 95\%.

Devido a impossibilidade de assinatura do Termo de Consentimento Livre e Esclarecido (TCLE) nos participantes da pesquisa, foi assinado um Termo de Confidencialidade visando preservar a privacidade das informações coletadas por análise documental. O estudo originou-se da dissertação Grau de complexidade de cuidados de enfermagem em readmissão hospitalar na oncologia clínica de câncer de mama ${ }^{(11)}$. Foi aprovado pelo Comitê de Ética em Pesquisa do Instituto Nacional do Câncer sob o parecer registrado no CAAE: 53075716.2.3001.5274.

\section{口ESULTADOS}

Verificou-se que 08 pacientes com câncer de mama readmitidos em 30 dias após a alta (7,4\%) foram categorizados no grau de complexidade de cuidados de enfermagem mínimos, 22 em cuidados intermediários (20,4\%), 39 em cuidados semi-intensivos $(36,1 \%)$ e a mesma quantidade, 39 $(36,1 \%)$, em cuidados de enfermagem intensivos (Gráfico 1).

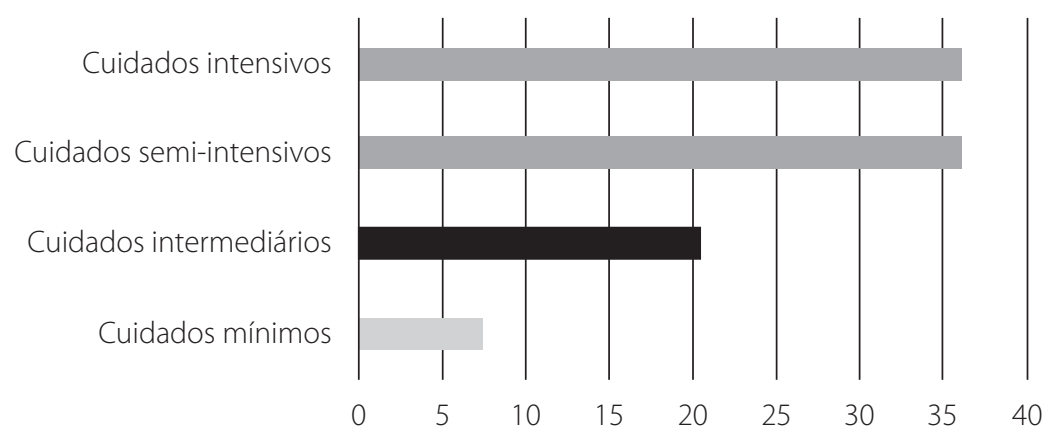

Gráfico 1 - Percentual de pessoas com câncer de mama readmitidas na unidade de internação de oncologia clínica segundo o grau de complexidade de cuidados de enfermagem. Rio de Janeiro- RJ, 2015 Fonte: Dados da pesquisa, 2017. 
A partir da análise estatística dos dados, conforme a Tabela 1 não foi encontrada significância estatística entre as características sociodemográicas e o grau de complexidade, bem como entre as características da rede assistencial e o grau de complexidade de cuidados de enfermagem.

Tabela 1 - Distribuição das características sociodemográficas, rede assistencial, tempo de matrícula e sua relação com grau de complexidade de cuidados de enfermagem de pessoas readmitidas na oncologia clínica ( $n=108)$. Rio de Janeiro - RJ, 2015

\begin{tabular}{|lllll|}
\hline Variável & $\begin{array}{l}\text { Cuidado mínimo/ } \\
\text { intermediário }\end{array}$ & $\begin{array}{l}\text { Cuidado semi-intensivo/ } \\
\text { intensivo }\end{array}$ & Total & Valor de p \\
\hline $\mathbf{n}(\%)$ & $\mathbf{n}(\%)$ & $\mathbf{n}(\%)$ & \\
\hline
\end{tabular}

\section{Faixa etária}

\begin{tabular}{lllll}
\hline Menor ou igual a 50 anos & $9(8,3)$ & $29(26,9)$ & $38(35,2)$ & $0,484^{\mathrm{a}}$ \\
Maior que 50 anos & $21(19,4)$ & $49(45,4)$ & $70(64,8)$ & \\
\hline Estado civil & & & & \\
\hline Com companheiro & $16(15,1)$ & $31(29,2)$ & $47(44,3)$ & $0,242^{\mathrm{a}}$ \\
Sem companheiro & $14(13,2)$ & $45(42,5)$ & $59(55,7)$ & \\
\hline Escolaridade & & & $90(83,3)$ & $0,102^{b}$ \\
\hline Ensino Fundamental e Médio & $23(21,3)$ & $67(62)$ & $16(14,8)$ & \\
Escolaridade Superior & $5(4,6)$ & $11(10,2)$ & $2(1,9)$ & \\
Escolaridade Analfabeto & $2(1,9)$ & $0(0)$ & $52(48,1)$ & \\
\hline Ocupações - CNAE' & & & $49(45,4)$ & $0,889^{b}$ \\
\hline Ocupações grupos CNAE & $15(13,9)$ & $37(34,3)$ & $7(6,5)$ & \\
Ocupação do lar & $14(13,0)$ & $35(32,4)$ & & \\
Aposentado/pensionista & $1(0,9)$ & $6(5,6)$ & $23(27,7)$ & \\
\hline Renda & & $15(18,1)$ & $44(53,0)$ & $0,639^{b}$ \\
\hline$<1$ salário mínimo & $8(9,6)$ & $33(39,8)$ & $11(13,3)$ & \\
1 a 2 salários mínimos & $11(13,3)$ & $9(10,8)$ & $5(6,0)$ & \\
3 a 5 salários mínimos & $2(2,4)$ & $3(3,6)$ & & \\
$<5$ salários mínimos & $2(2,4)$ & & & \\
\hline
\end{tabular}

Fonte: Dados da pesquisa, 2017.

Legenda: * 0 \% em n (\%) é a porcentagem em relação ao total da população do estudo.

Teste Qui Quadrado de Pearson ${ }^{\mathrm{b}}$ Teste Exato de Fisher

${ }^{1}$ CNAE: Classificação Nacional de Atividades Econômicas

Teste Qui Quadrado de Pearson ${ }^{\mathrm{b}}$ Teste Exato de Fisher

${ }^{1}$ CNAE: Classificação Nacional de Atividades Econômicas

Conforme mostra-se na Tabela 2, só foi encontrada significância estatística em algumas variáveis relacionadas às características da história clínica as pessoas com câncer de mama readmitidas na oncologia clínica. Destacam-se àquelas com significância estatística: estadiamento $(p=0,037)$, performance status $(p<0,001)$, comorbidades $(p=0,019)$, hipertensão arterial $(p=0,015)$ e sem comorbidades $(p=0,10)$.

As possíveis influências no desfecho conforme os dados supracitados são aquelas que ocorrem se as variáveis forem analisadas separadamente, ou seja, sem interferência de umas sobre as outras.

Na análise multivariada, considerando as interações das covariáveis e o desfecho grau de complexidade de cuida- dos de enfermagem, algumas associações não se mantiveram estatisticamente significantes (Tabela 3).

No modelo multivariado, ap enas o PS $(p<0,001)$ e a hipertensão arterial $(p=0,024)$ se mantiveram estatisticamente associados ao grau de complexidade de cuidados de enfermagem. Pode-se inferir ainda que as mulheres com câncer da mama readmitidas na oncologia clínica com PS 1 ou 2 têm 88\% (odds=0,122) menos chance de serem classificadas no grau de complexidade de cuidados semi-intensivos e intensivos. Esse mesmo grupo de muIheres quando portadoras de hipertensão arterial tem 56\% (odds $=3,562$ ) mais chance de ser classificado em cuidados de enfermagem semi-intensivos e intensivos. 
Tabela 2- Distribuição das características da história clínica e da causa da readmissão hospitalar em relação ao grau de complexidade de cuidados de enfermagem de pessoas readmitidas na oncologia clínica (n=108). Rio de Janeiro - RJ, 2015

\begin{tabular}{|c|c|c|c|c|}
\hline \multirow[b]{2}{*}{ Variável } & \multicolumn{4}{|c|}{ Grau de complexidade } \\
\hline & $\begin{array}{l}\text { Cuidado mínimo/ } \\
\text { intermediário }\end{array}$ & $\begin{array}{l}\text { Cuidado semi-intensivo/ } \\
\text { intensivo }\end{array}$ & Total & $\begin{array}{l}\text { Valor de } \\
\text { p }\end{array}$ \\
\hline & n $(\%)^{*}$ & n $(\%)^{*}$ & n $(\%)^{*}$ & \\
\hline \multicolumn{5}{|c|}{ Nível de atenção da rede que ocorreu diagnóstico do câncer de mama } \\
\hline Primário & $10(15,6)$ & $23(35,9)$ & $33(51,6)$ & \multirow{2}{*}{$0,920^{\mathrm{a}}$} \\
\hline Secundário e Terciário & $4(6,3)$ & $27(42,2)$ & $31(48,4)$ & \\
\hline \multicolumn{5}{|c|}{ Intervalo de readmissão hospitalar } \\
\hline Até 10 dias após a alta & $16(14,8)$ & $33(30,6)$ & $49(45,4)$ & \multirow[t]{2}{*}{$0,303^{a}$} \\
\hline Entre 11 e 30 dias após a alta & $14(13)$ & $45(41,7)$ & $59(54,6)$ & \\
\hline \multicolumn{5}{|l|}{ Estadiamento } \\
\hline$\|$ & $3(2,9)$ & $2(1,9)$ & $5(4,8)$ & \multirow[t]{3}{*}{$0,037^{b}$} \\
\hline III & $10(9,5)$ & $17(16,2)$ & $27(25,7)$ & \\
\hline IV & $15(14,3)$ & $58(55,2)$ & $73(69,5)$ & \\
\hline \multicolumn{5}{|l|}{ Performance status na readmissão } \\
\hline$P S^{1}-1$ e 2 & $13(12)$ & $8(7,4)$ & $21(19,4)$ & \multirow[t]{2}{*}{$<0,001^{a}$} \\
\hline$P S^{1}-3$ e 4 & $17(15,7)$ & $70(64,8)$ & $87(80,6)$ & \\
\hline \multicolumn{5}{|l|}{ Comorbidades } \\
\hline Hipertensão arterial & $8(7,4)$ & $41(38)$ & $49(45,4)$ & $0,015^{a}$ \\
\hline Diabetes & $4(3,7)$ & $8(7,4)$ & $12(11,1)$ & $0,734^{b}$ \\
\hline Hipotireoidismo & $1(0,9)$ & $6(5,6)$ & $7(6,5)$ & $0,671^{b}$ \\
\hline Insuficiência cardíaca congestiva & $1(0,9)$ & $4(3,7)$ & $5(4,6)$ & $1,000^{b}$ \\
\hline Insuficiência Renal & $0(0)$ & $2(1,9)$ & $2(1,9)$ & $1,000^{\mathrm{b}}$ \\
\hline Asma & $1(0,9)$ & $1(0,9)$ & $2(1,9)$ & $0,480^{b}$ \\
\hline Sem comorbidades & $16(14,8)$ & $21(19,4)$ & $37(34,3)$ & $0,010^{a}$ \\
\hline Outras comorbidades ${ }^{2}$ & $1(0,9)$ & $3(2,8)$ & $4(3,7)$ & $1,000^{b}$ \\
\hline
\end{tabular}

Fonte: Dados da pesquisa, 2017.

Legenda: * $0 \%$ em n (\%) é a porcentagem em relação ao total da população do estudo.

aTeste Qui Quadrado de Pearson bTeste Exato de Fisher 'PS: Escala de Performance status ${ }^{2}$ Traço Falciforme, Vitiligo, Estenose pilórica e Transtorno psiquiátrico.

Tabela 3- Modelagem de Regressão logística binária das possíveis variáveis associadas ao grau de complexidade de cuidados de enfermagem das pessoas com câncer de mama readmitidas na oncologia clínica (n=108). Rio de janeiro - RJ, 2015

\begin{tabular}{|c|c|c|c|c|}
\hline \multirow{2}{*}{ Variáveis } & \multirow{2}{*}{ p-valor } & \multirow{2}{*}{ Exp B (odds) } & \multicolumn{2}{|c|}{ Intervalo de confiança } \\
\hline & & & Inferior & Superior \\
\hline Performance status & $<0,001$ & $0,122^{1}$ & 0,048 & 0,424 \\
\hline Hipertensão arterial & 0,024 & $3,562^{2}$ & 1,245 & 10,658 \\
\hline Sem comorbidades & 0,476 & $*$ & * & * \\
\hline Estadiamento & 0,435 & * & * & * \\
\hline Escolaridade & 0,844 & $*$ & $*$ & * \\
\hline
\end{tabular}

Fonte: Dados da pesquisa, 2017.

Legenda: *Variáveis foram removidas da modelagem de regressão durante a execução por insignificância estatística.

${ }^{1}$ Razão de chance se mostra como fator de proteção, indicando 88\% menos chance da primeira categoria do PS (PS 1 e 2) ser classificada na última categoria do grau de complexidade de cuidados de enfermagem (Cuidados semiintensivos e intensivos).

${ }^{2}$ Razão de chance se mostra como fator de risco, indicando $56 \%$ mais chance da primeira categoria da Hipertensão (possuir hipertensão) ser classificada na última categoria do grau de complexidade de cuidados de enfermagem (Cuidados semi-intensivos e intensivos). 


\section{口 DISCUSSÃO}

Os graus de complexidade que predominaram na maioria das pessoas readmitidas foram cuidados semiintensivos $(36,1 \%)$ e cuidados intensivos $(36,1 \%)$, ambos com frequências similares. Esse resultado coaduna com os achados do estudo realizado num hospital universitário de Porto Alegre estado do Rio Grande do Sul ${ }^{(12)}$ onde 51,3\% dos pacientes internados foram classificados em cuidados semi-intensivos, 32\% em cuidados intermediários e 14,4\% em cuidados intensivos.

O número de internações por pacientes demonstra, muitas vezes, que esses demandam uma assistência baseada em cuidados mais complexos ${ }^{(7)}$. Portanto, é possível inferir que pessoas em situação de readmissão hospitalar requerem cuidados em maior número e/ou com maior grau de complexidade.

Cabe ressaltar que pelo cenário do estudo se tratar de uma unidade de internação, assim como em outras publicações, não se esperava encontrar uma predominância das classificações cuidados semi-intensivos e intensivos, pois tal setor não tem facilmente disponíveis recursos humanos, materiais e tecnológicos necessários a esse tipo de demanda.

O baixo índice de pessoas classificadas com cuidados mínimos $(7,4 \%)$ no estudo em questão instiga uma reflexão acerca do cenário do estudo e da mudança no perfil de pacientes internados em unidades de internações de instituições de referência para tratamento de doenças crônicas ${ }^{(12)}$. A dificuldade em obter um leito em Unidade de Terapia Intensiva (UTI) acaba demandando que pessoas com agravamento do quadro clínico ou em condições complexas permaneçam em unidades de internação. Bem como a predominância de pessoas com estadiamento avançado da doença, sem possibilidades terapêuticas para cura, também podem ser um dos fatores facilitadores para que pessoas com grau de complexidade de cuidados semi-intensivos e intensivos permaneçam em unidades de internação e não sejam encaminhadas para instituição ou setor especializado em cuidados paliativos.

Esse perfil de grau de complexidade de cuidado de enfermagem encontrado emerge a necessidade de uma reavaliação da alocação de recursos necessários ao atendimento das pessoas com câncer da mama readmitidas na oncologia clínica. A demanda dessa clientela carece de recursos humanos, materiais e tecnológicos especializados para cuidados semi-intensivos e intensivos, condições indispensáveis para assistência de enfermagem contínua, segura e qualidade ${ }^{(7,12)}$.

A caracterização do grau de complexidade de cuidados de enfermagem a partir de um SCP pode interferir no gerenciamento do cuidado(13). Ela é capaz de facilitar a identifica- ção das reais necessidades de saúde das pessoas internadas, auxiliar no gerenciamento de custos, embasar o dimensionamento e a alocação de recursos humanos de enfermagem, bem como recursos materiais e insumos necessários para o processo assistencial. Tais autoras relataram também que esse sistema tem sido utilizado de diversas formas em âmbito internacional, por exemplo: Na Suécia, o SCP designado como ZEBRA calcula o custo de pessoal de enfermagem por dia em substituição aos custos fixos padronizados; já na Finlândia, o instrumento RAFAELA, tem sido utilizado para diversas finalidades, como para mensurar a utilização e custos de profissionais de enfermagem no processo de cuidar, auxiliar no processo de tomada de decisão para alocação de recursos humanos, mensurar sobrecarga de trabalho associada a assistência prestada ao paciente.

Uma pesquisa sobre o uso do SCP RAFAELA examinando as taxas de mortalidade hospitalar, verificou que o índice de mortalidade hospitalar aumentou quando a carga de trabalho de enfermagem foi além do nível ideal e também quando houve falta de pessoal ${ }^{\left({ }^{(14)}\right.}$. Mediante a isso, pode-se dizer que o grau de complexidade elevado, pode aumentar o nível de sobrecarga dos profissionais de enfermagem, influenciar negativamente na qualidade da assistência e, consequentemente, aumentar o risco de mortalidade das pessoas internadas.

Verificou-se nos resultados também que houve diferença no grau de complexidade de cuidados de enfermagem em relação ao estadiamento $(p=0,037)$ e a presença do estádio IV $(p=0,032)$ entre as pessoas com câncer da mama readmitidas na oncologia clínica.

O prognóstico da doença nos casos de estadiamento avançado é pior e sua sobrevida é menor ${ }^{(15)}$. A ocorrência de diferença no grau de complexidade de acordo com o estadiamento demonstra que o estádio da doença pode influenciar o grau de complexidade de cuidados de enfermagem de pacientes com câncer da mama em readmissão hospitalar. Considera-se que a redução de casos de readmissão pode indicar qualidade assistencial, melhorar a qualidade de vida das pessoas e também reduzir custos para os serviços de saúde. Então, pode-se dizer que a identificação dos estadiamentos dos indivíduos internados, a inclusão dessa informação e suas peculiaridades na identificação de problemas relacionados ao seu processo saúdedoença podem facilitar a elaboração de um planejamento assistencial e, consequentemente, impactar nos cuidados prestados a esses indivíduos.

A classificação de pacientes é uma ferramenta de gestão de cuidado, subsidiando tanto a assistência indireta, com a previsão de recursos, organização do ambiente e sistematização de atividades, quanto a assistência direta voltada ao 
atendimento propriamente dito e aos cuidados prestados para atender as necessidades de saúde das pessoas.

O avanço da doença, o prognóstico desfavorável e até os episódios de readmissões decorrentes de complicações devem ser manejados com a meta de se trabalhar em torno da sobrevida, proporcionando a essas pessoas cuidados especializados, conforto, qualidade de vida, dignidade e alívio dos seus sinais e sintomas da melhor forma possível.

Os resultados mostraram também a presença de diferença no grau de complexidade de cuidados de enfermagem de acordo com a performance status $(p<0,001)$, hipertensão arterial $(p=0,015)$ e ausência de comorbidades $(p=0,010)$ das mulheres com câncer da mama readmitidas na oncologia clínica.

No modelo multivariado, apenas o PS $(p<0,001)$ e a hipertensão arterial $(p=0,024)$ se mantiveram estatisticamente associados ao grau de complexidade de cuidados de enfermagem. Isso indica que elas são variáveis associadas ao desfecho, ou seja, as mesmas, de formas independentes, podem indicar se o grau de complexidade de cuidados de enfermagem é mais elevado ou não.

As mulheres com câncer da mama readmitidas na oncologia clínica com PS 1 ou 2 têm mais chance de serem classificadas em cuidados mínimos e intermediários. Já quanto as portadoras de hipertensão arterial, há maior chance destas requerem cuidados de enfermagem semiintensivos e intensivos.

Um estudo com mulheres com câncer de mama e ginecológicos avançados constatou que a performance status é uma variável preditora para sobrevida dessas mulheres. Quanto menor sua capacidade funcional obtida pela escala de performance status, menor será sua sobrevida ${ }^{(16)}$. Já um outro estudo com pessoas internadas em UTI mostrou que as mais graves e com menores chances de sobrevida demandaram maior carga de trabalho da equipe de enfermagem, ou seja, maior grau de complexidade de cuidados de enfermagem ${ }^{(17)}$.

De acordo com o Manual de Cuidados de Paliativos, a avaliação de pacientes internados é fundamental para vigilância da curva evolutiva da doença. Esta é capaz de fundamentar a tomada de decisão, previsão de prognóstico e diagnóstico de terminalidade e uma das ferramentas para essa avaliação é a escala de performance status(18).

A escala de PS é extremamente útil para planejar o tratamento e o cuidado de pacientes oncológicos. A mesma pode ser aplicada diariamente em unidades de internação, emergências e até consultas ambulatoriais. Sua aplicação é mais simplificada que o instrumento completo do SCP de Perroca ${ }^{(8)}$, sendo composto por apenas o indicador de performance clínica obtido pelas respostas de 5 escores.
A avaliação da capacidade funcional do paciente com câncer da mama readmitido na oncologia clínica por meio do PS poderia permitir, dentre outras, identificar quais pessoas demandariam maior grau de complexidade de cuidados de enfermagem. Isso embasaria uma tomada de decisão rápida pela equipe multiprofissional acerca de recursos a serem alocados, principalmente humanos.

Entretanto, a aplicação dessa escala de forma única na assistência de enfermagem, não permite a identificação das necessidades de cuidados da clientela a ser assistida. Ela não informa os problemas que as pessoas com doença oncológica podem ter em todas as dimensões da saúde que a neoplasia acomete, sejam elas biológica, psicossocial ou espiritual. Há, portanto, necessidade de investigar outros domínios que contemplariam o estabelecimento do cuidado integral e humanizado a essa clientela.

A hipertensão arterial (HAS) pode ser frequentemente decorrente de medicações quimioterápicas as quais os pacientes são submetidos e que ocasionam toxicidade cardíaca. Pessoas em tratamento oncológico com drogas cardiotóxicas, medicações comuns nos protocolos quimioterápicos para câncer de mama, devem ser devidamente acompanhadas e monitoradas para se prevenir disfunções ventriculares e insuficiências cardíacas e, obter um diagnóstico precoce de qualquer anormalidade ${ }^{(19)}$.

A HAS também foi verificada nesse estudo como uma variável preditora independente para o grau de complexidade de cuidados de enfermagem. Vale destacar que que o Manual de Cuidados Paliativos coloca que o processo de tomada decisão em todos os pacientes oncológicos em estádio avançado da doença requer uma avaliação individualizada baseada nas suas comorbidades associadas ${ }^{(18)}$.

A sobrevida do câncer da mama feminina diminui à medida que o índice de comorbidade aumenta ${ }^{(15)}$. Pessoas com escores de comorbidade maior ou igual a 2, de acordo com a escala de Charlson usada no estudo supracitado, tinham aproximadamente 2 vezes mais chances de morrer do que aqueles que não as possui.

O manejo das comorbidades aliado ao tratamento para o câncer da mama é um desafio para a prática clínica. Este pode complicar a organização do cuidado, pois envolve a implementação de intervenções com objetivos em mais de uma doença. O cuidado integral da pessoa com câncer da mama envolve tanto a neoplasia como todos os elementos presentes no seu processo saúde-doença. 0 controle das comorbidades, seu tratamento e suas implicações no decorrer do processo da doença neoplásica podem diminuir o risco de morbidade e mortalidade, pois o mesmo é um preditor forte e importante para a sobrevida em câncer de mama ${ }^{(20)}$. 
A identificação das comorbidades e a inclusão dessa informação na elaboração do planejamento assistencial de enfermagem e nas discussões sobre os casos das pessoas internadas pela equipe multidisciplinar podem ser uma ferramenta valiosa para seu manejo e para evitar complicações evitáveis.

Durante a readmissão hospitalar, o indivíduo continua sendo portador de suas doenças de base, seja hipertensão, diabetes, cardiopatia ou depressão, e por isso, continua necessitando das terapêuticas destinadas a essas condições patológicas. Desconsiderar as tais pode desencadear descompensação de quadro clínico. Mesmo no alcance da resolutividade do motivo que justificou sua readmissão associado a neoplasia, seus antecedentes patológicos não desaparecem, muito pelo contrário, podem necessitar de um manejo mais complexo e até aumentar o risco de morte dessas pessoas por condições externas ao câncer.

As características clínicas e psicossociais das pessoas definem seu grau de complexidade de cuidados de enfermagem. Por meio deste é possível identificar a demanda de atenção dos pacientes em relação a equipe de enfermagem, prever recursos necessários, estimular a capacitação da equipe para que a mesma seja capaz de abordar o indivíduo em sua totalidade, compreendendo e prestando um cuidado humanizado e integral.

O índice de sobrecarga dos profissionais de enfermagem aumentou nos últimos anos devido à elevação da demanda sem o proporcional aumento dos recursos humanos em quantidade e qualidade adequadas para uma adequada assistência ${ }^{(12)}$. Nas últimas décadas, tem-se diminuído o tempo de permanência no hospital, pacientes recebem alta hospitalar com níveis mais altos de complexidade requerendo maior auxílio, conhecimento e capacidade para seu autocuidado em seu domicílio. A equipe de enfermagem tem tido menor tempo para elaborar planejamentos de alta com as devidas orientações que poderiam facilitar a continuidade do cuidado e até fazendo vínculos entre pontos da rede de atenção para que o indivíduo possa ser mais facilmente acompanhado na atenção primária.

É importante que a pessoa com câncer da mama compreenda a importância e como deve proceder quanto as terapêuticas que devem ser continuadas em seu domicílio após a alta hospitalar. O reconhecimento dos sinais de alerta para que a mesma procure um serviço de saúde especializado em oncologia antes que o problema piore é de extrema relevância.

A enfermeira deve realizar um gerenciamento do cuidado com etapas dentro do processo de enfermagem que envolva orientações para família e paciente acerca da: importância da continuidade da administração de medicamentos prescritos em seu domicílio; continuidade de seguimento com médico generalista na atenção primária, cardiologista ou endocrinologista em caso de presença de comorbidades; esclarecimentos quanto a possíveis complicações que poderão surgir nos primeiros dias de alta hospitalar e medidas necessárias ao seu manejo ainda no domicílio.

A tomada de decisão, instrumentalizada pelo grau de complexidade, se insere no gerenciamento do cuidado, tanto na assistência indireta quanto na assistência direta. A enfermeira oncológica que conhece as singularidades das pessoas as quais assiste, tem seu processo de trabalho pautado nas condições, estruturas e recursos indispensáveis para a dispensação de uma assistência de enfermagem oncológica segura e de qualidade.

\section{口 CONCLUSÃO}

O grau de complexidade dos pacientes com câncer de mama em readmissão hospitalar em até 30 dias na oncologia clínica caracterizou-se predominantemente semi-intensivo em 39 casos $(36,1 \%)$ e intensivo na mesma quantidade ao anterior com 39 casos (36,1\%). Seguidos por cuidados de enfermagem intermediários em 22 casos $(20,4 \%)$ e 08 pacientes em cuidados de enfermagem mínimos (7,4\%). Mesmo as readmissões hospitalares ocorrendo em unidade de internação, a predominância foi dos cuidados semi-intensivos e intensivos.

Dentre as distintas variáveis de aspectos socio demográficos e clínicos, apenas a capacidade funcional $(p<0,001)$ e 0 antecedente pessoal para hipertensão arterial $(p=0,024)$ mostraram-se associadas ao grau de complexidade de cuidados de enfermagem das pessoas com câncer da mama.

Conhecer o grau de complexidade de cuidado de enfermagem requerido por uma determinada clientela pode ser uma ferramenta valiosa na execução do gerenciamento do cuidado a ser prestado, principalmente, no âmbito da oncologia.

A classificação do grau de complexidade e o gerenciamento do cuidado na atenção oncológica facilitam que sejam cumpridos os princípios dos SUS e da Política de Atenção Oncológica no que tange a integralidade. Isto implica no subsídio da prestação de um cuidado integral, contínuo, compartilhado com paciente, família e comunidade e articulação nos distintos pontos da rede de atenção à saúde.

Uma assistência de enfermagem segura e de qualidade exige conhecimento de sua clientela e suas demandas, dos serviços de saúde os quais fazem parte, bem como dos recursos e condições indispensáveis para sua garantia.

O estudo teve como limitação, o fato dos dados terem sido obtidos por fonte secundária de informação. Dois indi- 
cadores da SCP da Perroca, o suporte emocional e a educação em saúde, apresentavam certo nível de subjetividade. Acredita-se que se coletados a partir de uma avaliação frente com a frente com a pessoa internada após anamnese e exame físico, poderiam ter seus escores modificados. Mas, apesar dessa limitação, esse é um dos poucos estudos no Brasil abordando a readmissão hospitalar na oncologia e relacionando o grau de complexidade de cuidados de enfermagem e o gerenciamento do cuidado em oncologia.

O aprofundamento dessa temática pode aumentar a sensibilidade dos tomadores de decisão para o impacto da qualidade da assistência no processo saúde doença da população, redução de custos para os serviços de saúde, ajudando a reduzir episódios de readmissões hospitalar e facilitando a descentralização da atenção oncológica para os distintos pontos da rede de saúde.

\section{口 REFERÊNCIAS}

1. Perroca MG, Jericó MA, Paschoal JV. Identificação de necessidades de cuidados dos pacientes com e sem uso de instrumento de classificação. Rev Esc Enferm USP. 2014;48(4):625-31.

2. Stewart BW, Wild CP (editors). World cancer report 2014. Lyon: IARC; 2014.

3. Oshiro ML, Bergmann A, Silva RG, Costa KC, Travaim IEB, Silva GBS, et al. Câncer de mama avançado como evento sentinela para avaliação do programa de detecção precoce do câncer de mama no centro-oeste do Brasil. Rev Bras Cancerol. 2014;60(1):15-23.

4. Haddad NC, Carvalho AC, Novaes CO. Perfil sociodemográfico e de saúde de mulheres submetidas à cirurgia para câncer de mama. Rev HUPE. 2015;14(supl. 1):28-35

5. Merli APD, Lopes ES, Machareli CA. Reinternações no Hospital Estadual Bauru: ocorrência e possíveis causas. Saúde Coletiva Digital. 2013;1(1):26-30.

6. Gavankar C, Gross C, Lilenbaum R, Chagpar AB. Factors associated with unplanned breast cancer readmissions [Abstract]. J Clinic Oncol. 2013;(31 supl):131.
7. Magalhaes AM, Riboldi CO, Guzinski C, Silva RC, Moura GM. Grau de dependência de pacientes em unidade de internação cirúrgica. Rev Bras Enferm. 2015;68(5):824-9.

8. Perroca MG. Desenvolvimento e validação de conteúdo da nova versão de um instrumento para classificação de pacientes. Rev Latino-Am Enfermagem. 2011;19(1):[9 telas].

9. West $\mathrm{H}$, Jin J0. Performance status in patients with cancer. JAMA Oncology. 2015;1(7):998.

10. Giuliano AE, Connolly JL, Edge SB, Mittendorf EA, Rugo HS, Solin L, et al. Breast cancer: major changes in the American Joint Committe on Cancer eight edition cancer staging manual. CA Cancer J Clin. 2017;67(4):290-303.

11. Silva, LG. Grau de complexidade de cuidados de enfermagem em readmissão hospitalar na oncologia clínica de câncer de mama [dissertação]. Rio de Janeiro (RJ): Universidade Federal do Rio de Janeiro; 2016.

12. 12. Silva KS, Echer IC, Magalhaes AM. Grau de dependência dos pacientes em relação à equipe de enfermagem: uma ferramenta de gestão. Esc Anna Nery. 2016;20(3):e20160060.

13. Abreu SP, Pompeo DA, Perroca MG. Utilização de instrumentos de classificação de pacientes: análise da produção do conhecimento brasileira. Rev Esc Enferm USP. 2014;48(6):1111-8.

14. Junttila JK, Koivu A, Fageström L, Haatainen K, Nykänen P. Hospital mortality and optimality of nursing workload: a study on the predictive validity of the RAFAELA Nursing Intensity and Staffing system. Int J Nurs Stud. 2016;60:46-53.

15. Shi R, Taylor H, McLarty J, Liu L, Mills G, Burton G. Effects of payer status on breast cancer survival: a retrospective study. BMC Cancer. 2015;15:211.

16. Rugno FC. Avaliação da fase de transição para cuidados paliativos exclusivos em pacientes com cânceres de mama e ginecológico avançados [dissertação]. Barretos (SP): Hospital de Câncer de Barretos; 2013.

17. Leite IR, Silva GR, Padilha KG. Nursing Activities score and demand of nursing work in intensive care. Acta Paul Enferm. 2015;25(6):837-43.

18. Academia Nacional de Cuidados Paliativos (BR). Manual de cuidados paliativos ANCP: ampliado e atualizado. Carvalho RT, Parsons HA (organizadores). 2 ed. Rio de Janeiro: ANCP; 2012.

19. Rocha RM, Schneider RS, Moreira I. Cardio-oncologia: onde estamos? Rev HUPE. 2013;12(Supl 1):93-9.

20. Ferreira DB, Mattos IE. Tendência da mortalidade por câncer de mama em mulheres no estado do Rio de Janeiro, Brasil, 1996-2011. Ciênc Saúde Coletiva. 2015;20(3):895-903.

Recebido: 05.02.2018

Aprovado: 31.07.2018
Lívia Gomes da Silva

E-mail: livia_nurse@yahoo.com.br 